\title{
Increasing efficiency in Grid by using glow worm Algorithm
}

\author{
Sedigheh Navaezadeh \\ Department of Computer \\ Science and Research Branch \\ Islamic Azad University \\ Khouzestan, Iran
}

\author{
Mehdi Sadeghzadeh \\ Member of Science Board of \\ Computer group in Azad \\ Islamic university of Mahshahr.
}

\author{
Ali Harounabadi \\ Member of Science Board of \\ Computer Group in Azad \\ Islamic university of Tehran \\ center.
}

\begin{abstract}
Nowadays, human beings encounter with hug data. Due to development of computer and detector technologies, few terabyte data has been produced. The analysis of these information require the sources whose financial costs cannot be afforded by any institution. In order to meet this demand, Grid computing has been considered as one of the most important fields of research. One of the important issues in grid environment is reliability in Grid service which must be studied. The study of Grid service helps systems' designers to create better systems with more reliability. Also, it helps the users to use Grid environment logically. The main purpose of Grid system is preparing services with high reliability and the least cost for many users as well as supporting collaborative jobs. On one hand, resources have important role in increasing and decreasing Grid application. If resources are limited, defilewii be the result; therefore, will decrease Gird efficiency will decrease. In this paper, swarm intelligence Algorithm has been applied for Grid load balance. Since Gird environment is dynamic, we use glowworm algorithm in sites in order to balance the load. The algorithm simulation results presented by MATLAB software showed that this algorithm can increase efficiency as well as Grid environment reliability.
\end{abstract}

Keywords: swarm intelligence algorithm, glowworm algorithm, Grid, load balance.

\section{INTRODUCTION}

Solving many problems in the field of engineering and other sciences requires using powerful computing resources. Computing Grid environments are suitable beds for solving problems which need long and difficult computing. Resources have been geographically distributed in this environment, but they can be logically considered as a unit source. Grid computing systems have been created as an important and modern field, and are different from common distributed systems. This is due to source subscription in large scales and innovative applicable programs with high efficiency. Grid computing system is a kind of distributed systems with a broad range. The main and special problem of Grid concept is resource subscription coordination, dynamic problem solving and multi- distributed programs.

Subscription in which Grid computing is involved is not only file primary exchange but also direct accessibility to computers, software data and other sources needing a broad spectra of problem solving strategies and resource subscription. Therefore, Grid computing reliability has been influenced by the relation existing between computing programs and important sources [1].

Since scheduling problem is related to NP-hard issue, we can use the assessment algorithm to solve it. Among these algorithms, genetic algorithm (GA) is more common, so it has been used in this paper.

GA presents new solutions through mixing the best options and existing solutions randomly. In this algorithm, the function called intersection has been used for general quest among the solutions by means of changing a part of 2 chromosomes randomly. Another important local research operator is mutation in which random change of one gene in a chromosome has been used and applied. Whole process that has been repeated several times is called propagation or reproduction [2]. The advantages of computing Grid are as the following: sources, parallel computing ability, creating source and virtual organization, using source, reliability, management and more source availability [3].

Since artificial intelligence algorithms are efficient in optimization, they have been considered as good options for solving the problems such as load balance in distributed system. The purpose of load balance is decreasing the rate of difference between the heaviest node and the lightest node in terms of load rate.

\section{LITERATURE}

Load Balance algorithm is divided into different groups. On one hand, these algorithms are divided into two groups; namely, static and dynamic. The difference of load balance in dynamic and static mode is that, in static mode, the decisions related to load balance are made in compiling time, while, in dynamic mode, decisions related to load balance are made in performing time. That is, in static mode, these decisions are made in the time of request for the source, but, in dynamic mode, the behavior of balancer varies according to the changes of parameters and policies [5].

Load balancing is divided into three groups: concentrated, non - concentrated and hierarchy. In concentrated method, all functions are scheduled by a scheduler, and scheduling operation is performed by the applicant source. In hierarchy method, the scheduler is organized in the hierarchy form [6]

In load balance of concentrated method, many studies have been conducted. In order to balance the load, genetic algorithm has been used by [5], and its Simulation result has been compared with Min - Max and Max - Min algorithm. In [2], a new genetic algorithm has been presented by using resource fault occurrence history (RFOH) for certain scheduling in computing Grid.

This strategy saves source fault occurrence history in Gird information server. GA is a general quest technique in which complex probable solutions called chromosomes have been applied and used. GA has created new solutions through mixing the best options among existing solutions randomly. The use of this information has decreased selection chance of the resources having more failure probability.

Simulation results showed that proposed strategy decreased total time of programs performance. One of glowworm algorithm advantages relating to other swarm intelligence algorithms is its constriction simplicity.

Firefly Algorithm is a type of algorithm obtained from nature and collective smart algorithm which presented by yang in 
2008. This algorithm is a modern technique based on collective behaviors and inspired from firefly social behaviors in the nature. Swarm intelligence is a type of artificial intelligence based on collective behaviors and neutralized and self-organized foundations. Fireflies generate rhythmic and short beams. Optical patterns of each firefly are different from others. Fireflies use these beams for two reasons: pairs attraction process, and attracting hunt. Moreover, these beams are used as a protective mechanism for fireflies. Due to rhythmic beams and rate of radiation and interval rate between light signals, two genders attract each other. Each particle of the firefly in multidimensional quest space is updated by dynamic absorption and based on the knowledge of firefly and its neighbors.

Firefly optimization algorithm can be stated as follows [7]:

* All fireflies are single- gender, and the factor of pairs attractiveness are considered regardless of their gender.

* Firefly $\mathrm{x}$ attracts all fireflies, and is attracted by all fireflies.

* Attractiveness is related to their glow, so in a pair of firefly, a worm with less light is attracted toward a worm with more light. Attractiveness power is related to their beam, and the light intensity decreases when the distance between two fireflies increases. If a firefly is not brighter than others, their movement will be performed randomly.

* Brighter firefly moves randomly (all fireflies can not attract them).

* Firefly brightness is determined by objective function value. Light intensity can be easily determined by target function.

* Firefly particles are randomly distributed in quest space according to above principles. There are two main parts in firefly algorithm: attracting firefly and moving toward the attracted firefly.

\subsection{General Form Of Firefly Algorithm}

General form of firefly algorithm has been shown in figure (1). As it can be observed in this figure, primary coordination and light intensity rate and the distance between firefly particles are firstly determined in quest area. In Quest procedure of firefly algorithm. Each firefly is individually compared with other fireflies. If the firefly has less light than the compared one, it will move toward the firefly with more light (the problem of finding maximum point), and due to this process, particles are centralized around a particle with more light. In the next generation of algorithm, if there is a particle with more light, then the particles will again move toward the particle with more light. Quest stages must be generated according to maximum number of repetitions. In this paper, in order to optimize the problem of load balance through using glowworm group intelligence algorithm, a solution has been presented. In this method, each node in the network is considered as a glowworm. Each glowworm tries to optimize its own existing load rate, and this work can be done by exchanging the load with other nodes.

\section{THE PROPOSED ALGORITHM}

The application of glowworm algorithm has been explained in previous sections. In the algorithm presented on the basis of swarm intelligence, all nodes in Grid system are considered as a solution for finding the most optimized mode.

The place of each node has been shown by the existing rate of light. In order to determine the attraction parameter for each node, its node light rate is compared with neighbor nodes. Each node always moves toward the best neighbor. This work can be done by attracting toward the neighbor or emitting more light from neighbor nodes when the rate of a node light is as same as the rate of neighbor nodes. The movement is performed randomly.

Since grid is a dynamic environment, solutions are always changing; therefore, in this method, there is no need to keep information and previous history like classic glow worm . In addition, no massive particles are required. In this research, parameter $\alpha, \beta$ and $\mathrm{r}$ are considered from 0 to 1 .

Particle Algorithm()

sourceLoad

while running

Do if job Queue. size>0

Then

Lighteload \% choose best Neighbor(entekhab avalin behtarin)

SecondLightestLoad \%\% choose SecondLightest

Neighbor(en entekhab dovomin behtarin)

TC (kamtarin hazine tebghe kamtarin faseleh)

threshold(tebghe avalin va dovomin)

while tc $>$ threshold

do

Submit jobs $\% \%(T C)$

sourceLoad $\% \%$ (currentNodeload)

velocity $\quad \% \%($ sourceload-lightestLoad)

Figure 1: Running Algorithm by using glow worm.

\subsection{Simulation Results}

After the proposed algorithm simulation in MATLAB environment, the results are compared with massive particles and genetic. The procedure of creating the network topology for the simulation is as follows:

At first, a minimum covering tree is structured, and then topology is obtained by adding the edges. In figure (2), time of the first job sending to the network and the last job are compared. The diagram showed the application of glowworm algorithm is more optimized than two other algorithms.

In figure (3), the difference between the lightest and heaviest nodes have been indicated in terms of the load (the load in glow worm shows its own light, and due to its similarity with massive particles, we have shown job comparison with this algorithm.). In figure (4), the effects of increasing number of jobs on time have been shown. This time involves sending time of the first job and running the last job in the network. 


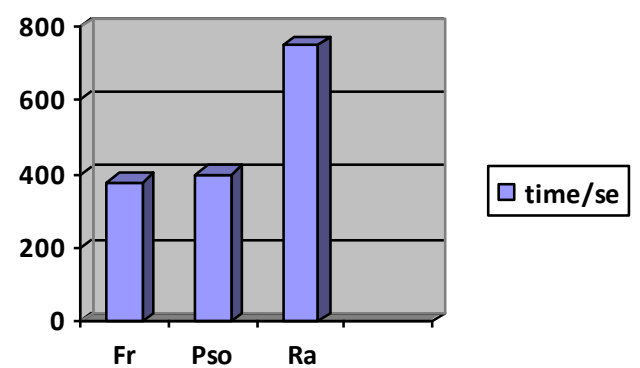

Figure 2: the advantage of running time in different algorithms

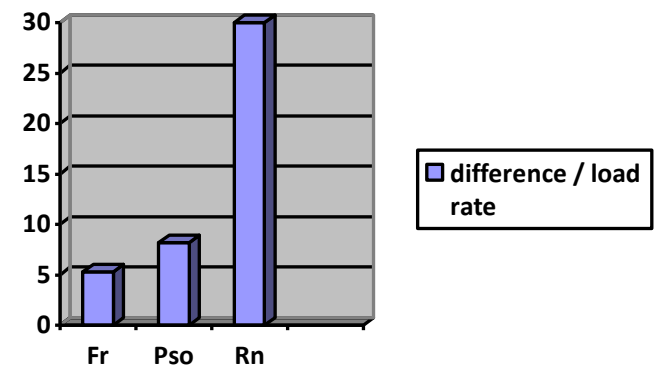

Figure 3: the difference between load rate of the worm with less light and the worm with more light.

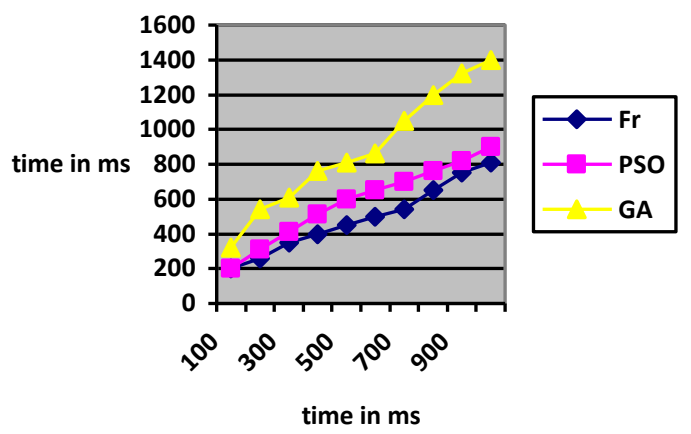

Figure 4: increasing the number of jobs along with increasing the number of jobs time.

\section{CONCLUSION}

In this paper, glow worm algorithm has been considered in order to balance the load in Grid. The results showed that this algorithm is more efficient than other algorithms when scheduling is considered, and there are different sources. Glow worm algorithm showed better results in terms of the running time. In addition, glowworm algorithm has been considered as the most appropriate methods for optimization problems.

\section{REFERENCES}

[1] Dai .Y.S, . Xie. M, Poh .K.L. ( 2010 ),.," Reliability Analysis of Grid Computing Systems", Department of Industrial and System Engineering, National University of Singapore.

[2] Mohammad Khanli. Leyli, Etminan Far . Maryam, Ghaffari . Ali. 2010," Reliable Job Scheduler using RFOH in Grid Computing", Journal of Emerging Trends in Computing and Information Sciences CIS Journal. All rights reserved.

[3] Cao. J, Spooner. D. P, Jarvis. S. A, Nudd.v.,( 2005)._Grid load balancing using intelligent agents. Future_Gener. Comput. System.

[4] Grosan. C, Abraham. A, and Helvik. B. ( 2007).Multiobjective evolutionary algorithms for scheduling jobs on computational grids.ADIS InternationalConference, Applied Computing, Salamanca,Spain, Nuno Guimares and Pedro Isaias (Eds).

[5] Subrata. R., Zomaya. A. Y, and. Landfeldt. B( 2007). Arti cial life techniques for load balancing in computational_grids. J. Comput. Syst. Sci.,

[6] J. K. J and. Eberhart. R. C,( 2001). Swarm Intelligence._Morgan Kaufmann Publishers

[7] Hassanzadeh, T. Meibodi, M, 1390. Presenting Improved Glowworm Algorithm for Optimization in Static Environment. The Fifth Conference of Datamining in Iran. Amirkabir University, Iran:Tehran. 